\title{
Physical Requirements for Flares in Stars
}

\author{
Bernhard Haisch \\ Lockheed Martin Solar $\&$ Astrophysics Laboratory, 3251 Hanover Street, \\ B/252, Dept. L941, Palo Alto, CA 94304
}

\begin{abstract}
A wide range of stellar analogs of solar X-ray flares has been observed. During the maximum of the solar activity cycle, one or two $\mathrm{M}$ class flares peaking at $L_{x} \sim 10^{26} \mathrm{erg} \mathrm{s}^{-1}$ in the GOES 1-8 $\AA$ passband take place on average every day on the Sun. Such run-of-the-mill events have been measured by ASCA on our nearest neighbor, Proxima Centauri, a dM5.5e flare star (Haisch, Antunes \& Schmitt 1995). At the other extreme, RS CVn systems and T Tauri stars have been observed to flare with peak luminosities of $L_{x} \sim \times 10^{32} \mathrm{ergs} \mathrm{s}^{-1}$ (Haisch \& Schmitt 1996 and references therein). Current wisdom has it that this wide range of flares spanning at least six order of magnitude (and another factor of 10-100 if one counts even lower level solar flares) on the young $\mathrm{T}$ Tauri stars, main sequence G, K and M stars, and in evolved RS CVn subgiants and giants can all be understood as versions of solar flares originating ultimately in a convectively-driven magnetic dynamo. There is evidence pointing to two distinct types of dynamo perhaps even in the Sun (Durney, Young and Roxburgh 1993): the $\alpha-\omega$ dynamo $^{1}$ generated in a rather thin boundary layer near - in some interpretations just below - the interface between a star's radiative core and convective envelope; and a turbulent distributed dynamo operative more or less throughout a convection zone. But what is one to make of the evidence for flares on $\mathrm{Be}$ stars (Smith, Robinson and Corbet 1998) where there is no subsurface convection to drive a dynamo? (Convective envelopes are thought to begin among the late-A spectral type stars, and steadily deepen for cooler stars.) This paper does not attempt to provide an answer, but it does attempt to outline the characteristics and requirements of magneticallypowered flaring.
\end{abstract}

\section{The Solar Flare from SMM to Yohkoh}

The Solar Maximum Mission launched in February 1980 was dedicated to studying the physics of solar flares. Rendered mostly inoperative by a simple blown fuse in 1981, the mission was the first to be repaired by an astronaut in orbit in 1984 , and functioned for the remainder of the decade. Ironically SMM reentered the atmosphere prematurely owing to intense solar flaring in 1989. This mission

\footnotetext{
${ }^{1}$ One frequently sees this referenced as $\alpha \Omega$ dynamo in the recent stellar literature.
} 
was more oriented toward spectroscopy than imaging: spectrographs measured ultraviolet emission, soft and hard X-rays and gamma rays. Flare-associated turbulent motions and upflows of coronal-temperature plasma at velocities up to nearly $1000 \mathrm{~km} \mathrm{~s}^{-1}$ were measured. SMM observations lent weight to what came to be a standard X-ray flare model of particle beams streaming along magnetic field lines, heating the chromospheric and upper photosphere and leading to the filling of magnetic loops with multi-million degree plasma. In this model impulsive hard X-ray bursts and optical flaring occur due to bremsstrahlung and heating respectively at the footpoints of coronal loops. The rapidly rising and gradually decaying soft X-rays are interpreted as thermal emission from loops filling with evaporating plasma. In fact, this is only part of the flare picture and does not directly address either the original source of the energy nor the energization process. Indeed, it is at odds with at least one empirical aspect of flares: that in many cases there is a modest rise in the soft X-ray emission prior to any hard X-ray bursts that are assumed to signal the onset of particle beams heating the solar surface at the loop footpoints.

The Yohkoh mission launched in August 1991 carries a grazing-incidence soft X-ray telescope (SXT), a hard X-ray Fourier imager and a bent crystal spectrometer (see Acton et al. 1992). The $\sim 2.5$ arcsec imaging capability of the SXT allowed a key observation to be made about flaring loops: many have a cusp at the top. A second crucial discovery is that in a significant number of flares there is persistent hard X-ray emission coming from somewhere within the cusp (above the classical loop top) that may last throughout the flare (Masuda et al. 1994). Aschwanden et al. (1996) find that the hard X-ray source is on average at a height of 1.4 times the height of the loop, based on analysis of timing of hard X-ray pulses vs. energy from which a differential time-of-flight of electrons as a function of energy can be inferred, hence a distance.

The Yohkoh observations have led to the proposal by Shibata et al. (1995) of a unified flare model which is an updated version of the classical two-ribbon flare model proposed by Carmichael and by Sturrock in the 1960s, and further developed by Hirayama and by Kopp and Pneumann in the 1970s. The model is unified in that it is proposed that there is no fundamental difference after all between compact-loop flares and large two-ribbon flares, nor between single-loop flares and those involving the interaction of two loops.

Figure 1 is a simple schematic of the basic energization process: magnetic reconnection. If two loops (or indeed any magnetic flux tubes) of oppositelydirected polarity are forced into contact, a current sheet will form owing to Ampere's Law, $\nabla \times B=4 \pi \mathrm{J} / c$. Once a current is established, resistivity can convert the energy in the current into heating and mass motions. On the Sun the subsurface convective motions lead to convection cells at the surface which are on the order of $30000 \mathrm{~km}$ in size and which have a net flow pattern from the inside to the cell boundary. The largest loops tend to be anchored in the cell boundary regions (often referred to as the network owing to its appearance in chromospheric and transition region lines). But since the convection cells are not fixed in place, large-scale coronal loops are often made to collide with each other, resulting in current sheets. Yet another way to bring opposite magnetic polarities together is to have newly emerging flux tubes interact with existing loops. A third way involves footpoint motions within a loop. Parker (1988) investigated 


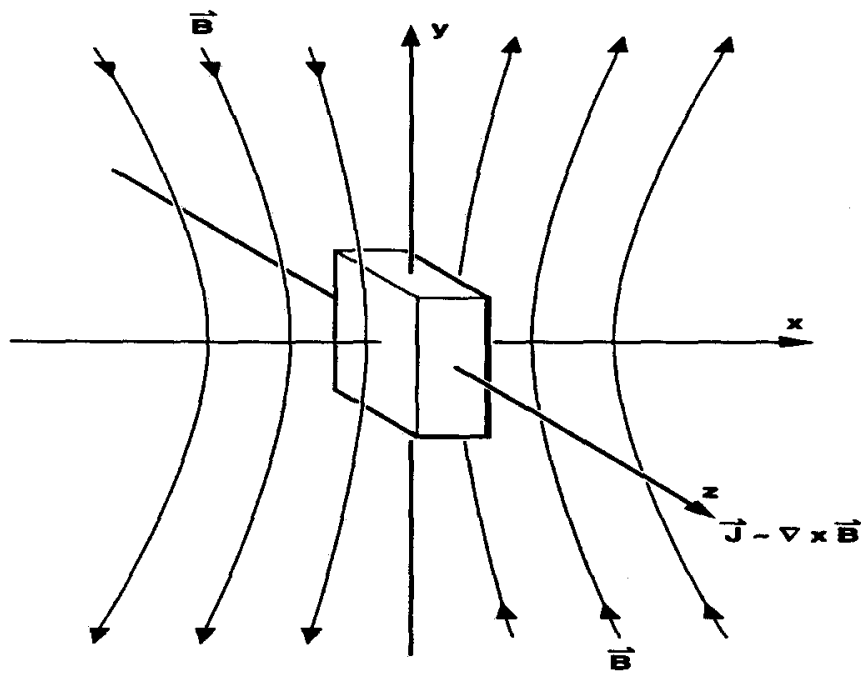

Figure 1. When oppositely-directed magnetic fields are forced together, a current sheet will be formed in which reconnection will take place.

current sheet formations within a loop leading to nanoflares occurring "as a consequence of random continuous motion of the footpoints of the field in the photospheric convection." He proposed this as a source of coronal heating, but from the perspective of the unified flare model, the fundamental ingredient of current sheet formation is the same for a nanoflare as for a huge flare. There is one more way for a current sheet to form: by instability of the magnetic field leading to the formation of a rising plasmoid. This is shown in Figure 2, the Shibata et al. (1995) model. The connection between a rising plasmoid creating a current sheet and formation of a current sheet via colliding oppositely-directed flux lines is that it appears to be a two-way street. If two loops collide, a plasmoid will almost certainly form above the collision-created neutral sheet. Figure 3 from Yokoyama and Shibata (1996) shows the similarity of the rising plasmoidinitiated flare and a loop collision-initiated flare. Because of the asymmetry in the latter, the analog of the plasmoid is a hot X-ray jet of the sort seen frequently by Yohkoh. As described by Yokoyama and Shibata (1995): "By the effect of finite resitivity, they (magnetic field lines) are cut and are reconnected with each other. The reconnection disconnects some of the highly stressed field lines in the emerging flux region and causes these field lines to fly outwards at one end as they try to straighten out. This whip-like motion accelerates (like a sling-shot) the plasma that lies on these field lines. At the same time, by Joule dissipation, magnetic energy is released as heat to increase the temperature of the plasma to a level when it emits X-rays. As a result, hot plasma is ejected from the reconnecting region, and may be observed as the X-ray jet." Their simulations indicate that not all of the sling-shot like ejected material is hot: the same 


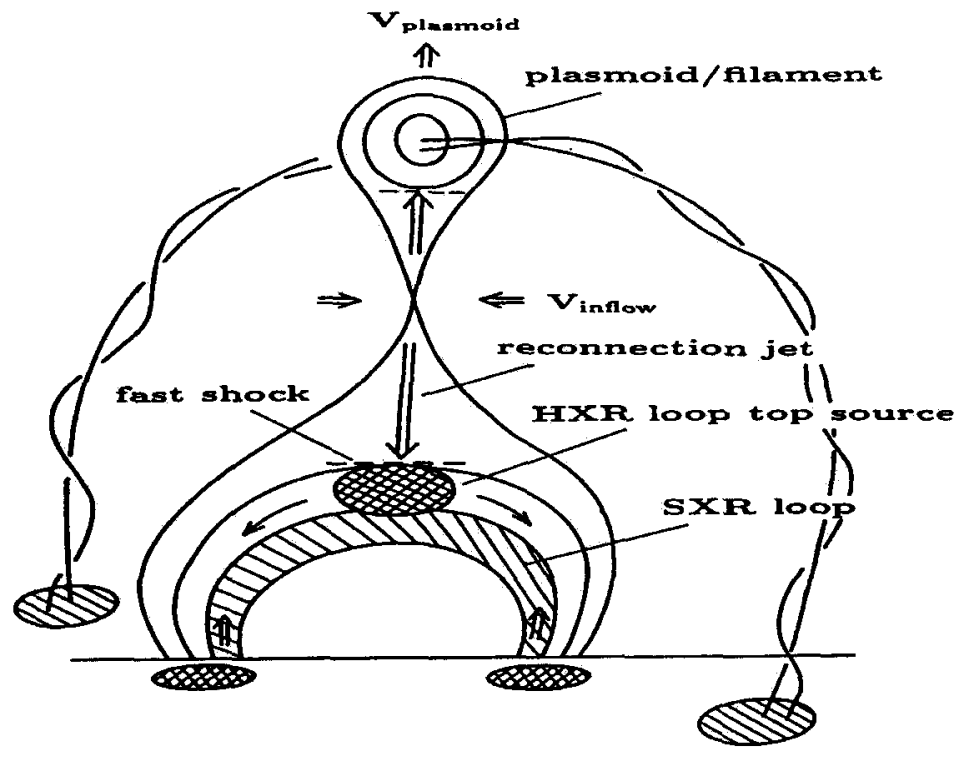

Figure 2. The Shibata et al. (1995) unified flare model (Shibata: with permission)

kind of reconnection process can also give rise to chromospheric-temperature $\mathrm{H} \alpha$ surges.

The plasmoid may or may not contain cool material prior to becoming unstable and flaring. If it does, it appears as a dark $H \alpha$ filament which transitions to an erupting prominence when the flare takes place. If there is no preflare concentration of cool material, the erupting plasmoid may still appear, but now as a coronal mass ejection (CME). CME's are frequently observed by the LASCO experiment onboard SOHO.

In the Shibata et al. model, "a high speed jet is created through the reconnection and collides with the loop top, producing fast-mode MHD shock, superhot plasma, and/or high energy electrons emitting hard X-rays." The initial stages of this process probably account for the "preflare" rise of soft X-rays which is often observed. This probably reflects activity in the low-density cusp region above the (soon-to-flare) loop prior to the onset of particle beams which then create the loop-filling evaporation of chromospheric material that constitutes the soft X-ray thermal phase. In this model the particle beam could be either a true beam consisting of electrons from the high energy tail end of a thermal distribution which undergo runaway acceleration owing to the reduction of the drag force at high thermal velocities, or the beam may be a superhot plasma (a few $100 \mathrm{MK}$ ).

\section{Which Dynamo?}

In a stellar convection zone one has forced motions due to turbulence, convection, magnetic buoyancy and differential rotation. Such motions of an electri- 

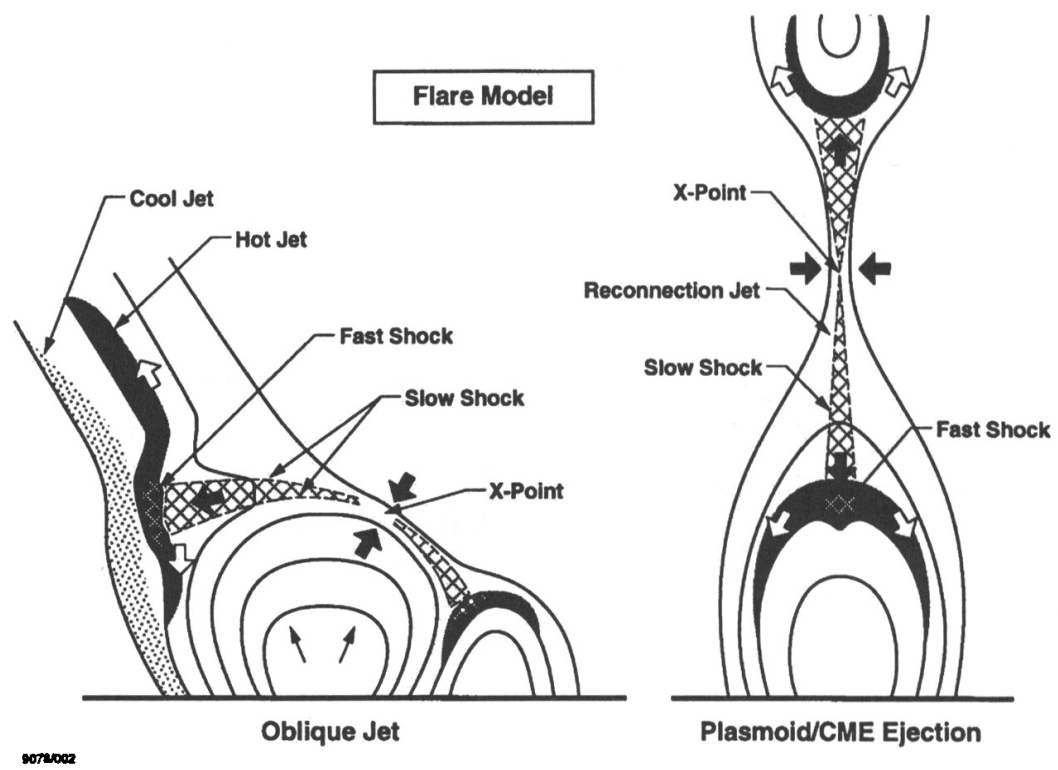

Figure 3. A loop-collision initiated flare (left) and a rising plasmoid flare (right) involve essentially the same processes, but in the former an X-ray jet is sometimes seen (adapted from Yokoyama and Shibata 1996; Shibata: with permission).

cally charged medium are virtually guaranteed to give rise to magnetic fields, the question being on what size scale and to what degree of amplification. The forced plasma motions create currents, the currents create magnetic fields, the time changing magnetic fields induce electric fields, which create currents. So long as these conditions work together, ampification will take place. It has been shown that such a purely convectively-driven dynamo - sometimes referred to as a turbulent-distributed or sometimes distributive dynamo - is feasible. Rosner (1980) first suggested that this kind of dynamo may be operative in fullyconvective stars. Durney et al. (1993) presented detailed theoretical arguments for the existence of this type of dynamo: "We show that the bulk of the stellar convection zone vigorously generates a small-scale turbulent magnetic field. Rotation, while not essential, increases the generation rate of this field. Thus, fully convective stars should have significant turbulent magnetic fields generated in their lower convection zones." Fully convective stars lie at the low-mass end of the main sequence. Fleming, Schmitt \& Giampapa (1995) found no change in the activity level of the very active $M$ dwarfs as one crosses the boundary around spectral type M5V between stars with a radiative/convective interface and those believed to be fully convective. Linsky et al. (1995) found a flare on the M8Ve star VB 10. The turbulent-distributed dynamo is a good candidate to explain the X-ray and flare activity of these very low mass $\left(\leq 0.3 M_{\odot}\right)$, fully convective $\mathrm{M}$ dwarfs. That this dynamo may also be operative in the Sun is 
suggested by the continued appearance of small-scale emerging magnetic flux on the Sun as evidenced by X-ray bright points even at the minimum of the cycle and at high latjtudes, for example. SOHO-MDI observations indicate that the "the magnetic flux on the Sun emerges uniformly over the surface during cycle minimum" (see comment by Title in Saar 1998) Indeed the ubiquitous "magnetic carpet" (Title and Schrijver 1998) may be generated by it.

Baliunas and Jastrow (1990) presented evidence - based on a chromospheric Ca II index - that a certain fraction (roughly one-fourth) of solar-like stars are in an extreme minimum activity state analogous to the Maunder Minimum of the Sun in the 17th century. Saar (1998) has now examined the relatively unchanging transition region and coronal emission of these stars (which he terms "Flat Activity" stars for lack of either cyclic behaviour or variability of any sort) and is proposing that the strength of such emission reflects the activity of a purely turbulent-distributed dynamo. Recently Stern (1998) suggested that since the most active stars (e.g. RS CVn systems) do not exhibit strong variability (outside of flares) nor evidence of cycles, that a distributed dynamo might be responsible for their activity. There is thus suggestive evidence for the reality of this type of dynamo from several directions.

The solar cycle, and presumably the bulk of solar flare activity, is believed to be driven by a more globally-organized shell or $\alpha-\omega$ dynamo, the $\omega$ referring to differential rotation and the $\alpha$ to a helicity effect in up and down motions of the magnetic field through the convection zone. (The reason that this dynamo is hypothesized to operate primarily in a radially-limited shell region has to do with the theoretical necessity to slow down the effects of magnetic buoyancy. Otherwise magnetic buoyancy would cause the field "to erupt much more rapidly than is consistent with observations" [Moffatt 1994].)

The solar surface differential rotation has been mapped out since the 19th century and this in combination with various theoretical considerations has produced estimates of the interior rotation as function of depth and latitude. Thanks to helioseismology observations by the Michelson-Doppler Imager onboard SOHO we now know the depth profile of solar rotation through the convection zone (Schou et al. 1998). As shown in Fig. 4, the solar differential rotation winds up (and concentrates) what is initially (at the start of a cycle) poloidal field and creates an E-W toroidal component. The critical radial differential rotation responsible for the " $\omega$-effect" has to lie somewhere near the interface between the radiative core and the convection zone, but it is not certain whether it is at the top of the radiative zone (Linsky et al. 1995) or the base of the convection zone. The extent of this critical region is on the order $0.03 R_{\odot}$. The new SOHO observations may help unravel this.

The other half of the effect was originally attributed to thermal convection, but is now thought to be due primarily to magnetic buoyancy (Brandenburg 1998). Since there is pressure equilibrium between the inside and the outside of a magnetic flux tube one has: $P_{\text {gas,int }}+B^{2} / 8 \pi=P_{\text {gas,ext. }}$. Given that the temperature is roughly the same, this means that the density is less inside the magnetic flux tube, hence the tube becomes buoyant. As it rises it is subject to Coriolis forces that give the tube a helical twist, the $\alpha$-effect. This is where the magnetic loops come from, some of which end up creating flares. 
Doppler imaging finds that there are spots on the polar regions of some RS CVn stars, T Tauri stars and a rapidly rotating $\mathrm{K}$ dwarf. This is consistent with the effect the Coriolis force would have on rapidly rotating stars (Valenti 1998; Schüssler and Solanki 1992). Johns-Krull and Valenti (1996) have made

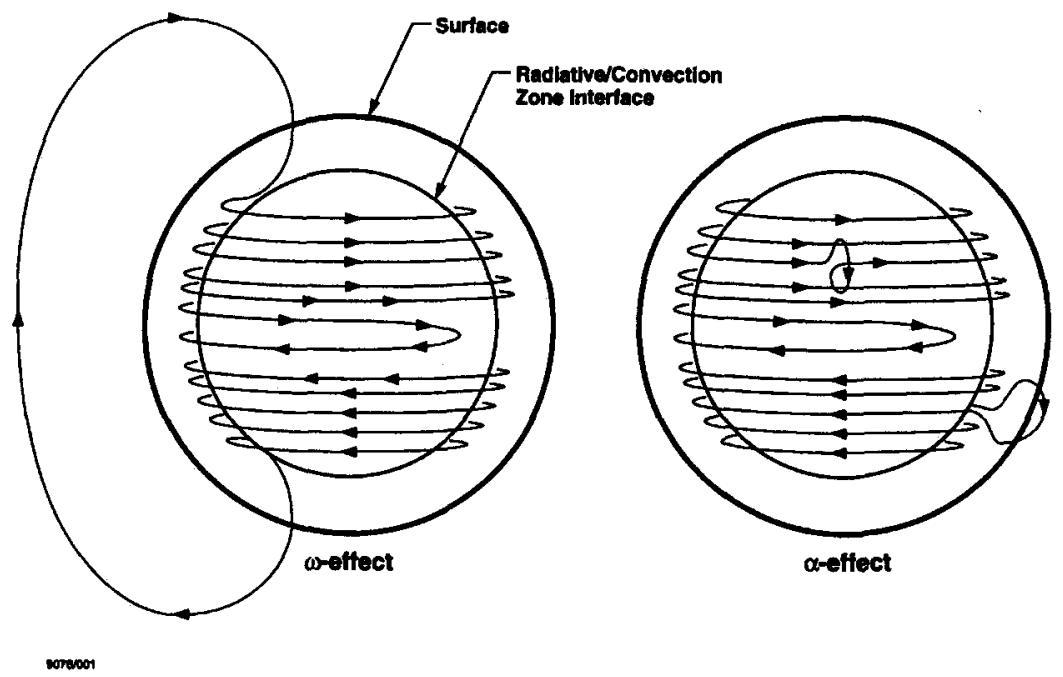

Figure 4. The shell or $\alpha-\omega$ dynamo. Differential rotation (the $\omega$-effect) winds up and concentrates poloidal field into toroidal field. Convection and magnetic buoyancy together with the Coriolis force (the $\alpha$-effect) create a helicity. When the flux tubes break through the surface a coronal loop appears.

measurements of the Zeeman-shifted $\sigma$ components of an Fe I line in two very active M4.5Ve flare stars. Through fitting of line profiles they arrive at estimates of 2-4 kG magnetic fields covering about 50 percent of the stellar surfaces. (For $\mathrm{G}$ and $\mathrm{K}$ dwarfs field strengths are typically a factor of two lower.) Now M4.5Ve stars are very close to being fully convective. Do these intense and widespread spot areas reflect some final maximum in shell dynamo activity concentrated in huge active regions, or might it be that the distributive dynamo is already at work and capable of creating a huge number of intense flux tubes scattered checkerboard-like across the entire stellar surfece?

\section{Other Flare Sites and Configurations}

\subsection{Interbinary magnetic fields:}

Flares in interbinary magnetic structures are a possibility. It was proposed by Uchida and Sakurai (1983) that magnetic field lines could extend from one star to another in close binary systems. There is as yet no direct evidence of this, but a recent 3D deconvolution of old Exosat data suggests an interconnecting loop-like structure in the TY Pyx system, a 3.2 day eclipsing RS CVn binary 
consisting of two G dwarfs with radii $R \sim 1.6 R_{\odot}$ and separation $d \sim 12 R_{\odot}$ (Pres, Siarkowski \& Sylwester 1995). Some aspects of interbinary flare-producing field configurations have been studied recently by Ferriera (1999). If two stars are close enough together their dipolar magnetospheres will squeeze each other in the region between the two stars. This can lead to the formation of a current sheet which can release energy directly. It can also lead to reconnection of field lines from one star to the other as shown in Figure 5. This in turn opens up the possibility of tapping additional sources of energy other than the magnetic field alone: viz, kinetic, gravitational and thermal. First of all, this would allow accretion of material onto one of the stellar surfaces releasing gravitational potential energy. As summarized by Ferreira: "After the accretion of this interbinary material, the base pressure at the interconnecting loops footpoints will in general be incompatible with a static equilibrium, and a siphon flow will be driven from one star to the other. This process can continue until the magnetic topology connecting the two stars is modified or until the loop base pressure changes and allows for a static equilibrium. These three associated processes; magnetic reconnection, accretion of interbinary plasma and accretion driven by a siphon mechanism, may together represent a good candidate to explain the very long duration flares observed in some binary systems... It is possible that the main source of energy for these flares is gravitational and centrifugal (associated with the material at the L1 point), and thermal (associated with the siphon flow) while the magnetic field... has a compartively small contribution."

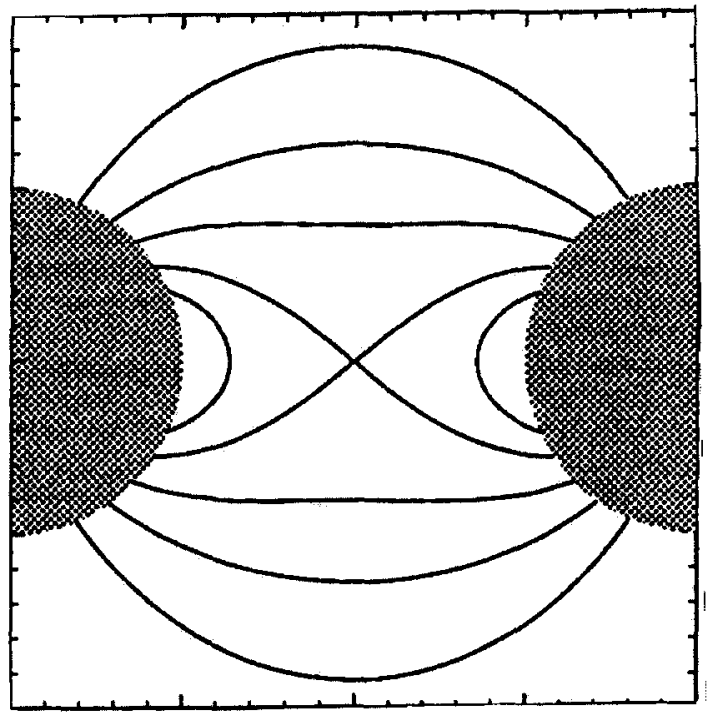

Figure 5. Reconnection of two squeezed dipole magnetospheres may result in this type of magnetic configuration (from Ferreira 1998; with permission). 


\subsection{A magnetospheric-tail flare:}

Yet another possible flare-producing situation exists in binaries if "a star that has a large-scale magnetic field interacts with the wind of the other star, forming a magnetospheric tail. This magnetotail can then release its energy and magnetic flux through flare-like events in many ways similar to the substorms that occur in the Earth's magnetosphere" (Ferreira 1998).

\subsection{Magnetically-stressed accretion disks:}

One can have a dynamo based on magneto-rotational instability instead of the solar-like magneto-convective instability. When a differentially rotating plasma is threaded by weak poloidal magnetic fields, it becomes unstable against axisymmetric perturbations (Balbus and Hawley 1992). However a purely toroidal field can also lead to instability (cf. Brandenburg et al. 1996 and references therein). Hawley, Gammie and Balbus (1996) carried out extensive simulations of these instabilities demonstrating how they "can amplify and sustain magnetic fields in the presence of dissipation, thus fulfilling the minimum criteria for a dynamo. This can lead to flares in stellar accretion disks ranging from protostars to neutron stars (Aly and Kuijpers 1990) to black holes (Volwerk, van Oss and Kuijpers 1993) Regarding a specific stellar flare mechanism for accretion disks, Hayashi, Shibata and Matsumoto (1996) carried out MHD simulations of interactions between the dipole magnetic field of a protostar and its accretion disk: "The closed magnetic loops connecting the central star and the disk are twisted by the disk. As the twist accumulates, magnetic loops expand and finally approach the open field configuration. A current sheet is formed inside the expanding loops. In the presence of resistivity, magnetic reconnection takes place in the current sheet. Outgoing magnetic islands and postflare loops are formed as a result of the reconnection." These magnetic islands are the same as the plasmoids in the Shibata et al. unified flare model. Similarly, heating to $10^{8} \mathrm{~K}$ is found in these simulations. The rotation of the disk creates helicity in place of the buoyancy-convection-Coriolis force mechanism operative in the solar $\alpha-\omega$ dynamo.

\subsection{Superflares:}

The term "superflare" is sometimes used to refer to the large, long-lived events (up to nine days in the case of CF Tuc; Kürster \& Schmitt 1995) on RS CVn stars with energies of $10^{37}-10^{38}$ ergs. The term could also be applied to events in which $L_{x} / L_{b o l}$ may be as high as several percent (cf. Haisch and Schmitt 1996 for several examples). Even such events can probably be explained as extreme examples of solar-like flares. But the event reported as a superflare by Montmerle and Grosso (1998) would be in a different category. They used the ROSAT HRI to observe X-rays from a Class I protostar (age $\sim 10^{5} \mathrm{yrs}$ ) deeply embedded in the $\rho$ Ophiuchi cloud. What they observed was a flare with $L_{x} / L_{b o l}>1$. They suggest that the Hayashi et al. type model of accretion-disk flaring or a flare involving magnetic interaction with a nearby companion - in which additional sources of energy could be tapped, as discussed above - would need to be invoked. 


\section{Conclusion}

Clearly there are many conditions under which stressed magnetic fields can give rise to flares on stars. But how does one create stressed magnetic fields on Be stars? Recently Tout and Pringle (1995) suggested a mechanism of dynamo activity that could be sustained even in these non-convective stars. Their argument is based on the possibility that there is a substantial shear component in the initial rapid rotation that accompanies these moderate-mass stars as they arrive on the main sequence. The effect which generates poloidal field from toroidal field is magnetic buoyancy in place of convection. A critical parameter is the efficiency of this process: if it is too efficient not enough shear energy will be trapped to sustain a dynamo.

These authors show that "for reasonable parameters, coronal activity can be sustained for $\mathrm{Ae} / \mathrm{Be}$ stars for an initial period until the shear energy has been exhausted." By "initial period" they mean no more than a few percent of the main sequence lifetime. After that the X-ray luminosity, and presumably also flare activity, would rapidly diminish. A comparison of fraction of coronally active Ae and Be stars with this activity lifetime fraction would be a useful first step in evaluating the possibility of this type of dynamo.

Acknowledgments. This work was supported by NASA contract NAS532071. BH thanks Myron Smith for the invitation to address this conference.

\section{References}

Acton, L.W. et al. 1992, Science, $258,618$.

Aly, J.J. \& Kuijpers, J. 1990, A\&A, 227, 473.

Aschwanden, M.J. et al. 1996, ApJ, 470, 1198.

Balbus, S.A. \& Hawley, J.F. 1992, ApJ, 400, 610.

Baliunas, S.L. \& Jastrow, R. 1990, Science, 348, 520.

Brandenburg, A. 1998, in Cool Stars, Stellar Systems and the Sun, (R. A. Donahue \& J.A. Bookbinder, eds.), ASP Conf. Series, 154, 173.

Brandenburg, A., Nordlund, A., Stein, R.F. \& Torkelsson, U. 1996, ApJ, 458, L45.

Durney, B., de Young, D. \& Roxburgh, I.W. 1993, Solar Phys., 145, 207

Ferreira, J.M. 1998, A\&A, 335, 248.

Fleming, T. A., Schmitt, J. H. M. M. \& Giampapa, M. S. 1995, ApJ, 450, 401.

Haisch, B., Antunes, A. \& Schmitt, J.H.M.M. 1995, Science, 268, 1327.

Haisch, B. \& Schmitt, J.H.M.M. 1996, PASP, 108, 113.

Hawley, J.F., Gammie, C. \& Balbus, S.A. 1996, ApJ, 464, 690.

Hayashi, M.R., Shibata, K. \& Matsumoto, R. 1996, ApJ, 468, L37.

Johns-Krull, C. M. \& Valenti, J. A. 1996, ApJ, 459, L95

Linsky, J.L. et al. 1995, ApJ, 4 55, 670.

Kürster, M. \& Schmitt, J.H.M.M. A\&A, 311, 211.

Masuda, S., Kosugi, T., Hara, H., Tsuneta, S. \& Ogawara, Y. 1994, Nature, $371,495$. 
Moffatt, H.K. in Lectures on Solar and Planetary Dynamos, (Cambridge Univ. Pres), 1994.

Montmerle, T. \& Grosso, N. 1998, in Cool Stars, Stellar Systems and the Sun, (R. A. Donahue \& J.A. Bookbinder, eds.), ASP Conf. Series, 154, CD1123.

Parker, E.N. 1988, ApJ, 330, 474.

Pres, P., Siarkowski, M. \& Sylwester, J. 1995, MNRAS, 275, 43.

Rosner, R. 1980, in Cool Stars, Stellar Systems and the Sun, (A. K. Dupree, ed.) SAO Rept. $389,79$.

Saar, S. 1998, in Cool Stars, Stellar Systems and the Sun, (R. A. Donahue \& J.A. Bookbinder, eds.), ASP Conf. Series, 154, 211.

Schou, J. et al. 1998, ApJ, 505, 390.

Schüssler, M. \& Solanki, S. 1992, A\&A, 264, L13.

Shibata, K. et al. 1995, ApJ, 451, L83.

Smith, M.A., Robinson, R. \& Corbet, R.H.D. 1998, ApJ, 503, 877.

Stern, R. 1998, in Cool Stars, Stellar Systems and the Sun, (R. A. Donahue \& J.A. Bookbinder, eds.), ASP Conf. Series, 154, 223.

Title, A. \& Schrijver, C.J. 1998, in Cool Stars, Stellar Systems and the Sun, (R. A. Donahue \& J.A. Bookbinder, eds.), ASP Conf. Series, 345.

Tout, C. A. \& Pringle, J.E. 1995, MNRAS, 272, 528.

Uchida, Y. and Sakurai, T. 1983, in IAU Coll. 71: Activity in Red-Dwarf Stars (P.B. Byrne \& M. Rodono, eds), 629.

Volwerk, M., van Oss, R.F. \& Kuijpers, J. 1993, A\&A, 270, 265.

Yokoyama, T. and Shibata, K. 1995, Nature, 375, 42.

Yokoyama, T. and Shibata, K. 1996, PASJ, 48, 353.

Valenti, J.A., Johns-Krull, C.M. \& N. Piskunov 1999, in Cool Stars, Stellar Systems and the Sun, (R. A. Donahue \& J.A. Bookbinder, eds.), ASP Conf. Series, 154, CD-1357.

\section{Discussion}

Rico Ignace: What are typical field strengths necessary for solar flares, and are there any basic scaling relations between field strengths and X-ray emission? B. Haisch: I am not aware of any significant differences between field strengths of non-flaring vs. flaring coronal loops. In both cases solar flux tubes have field strengths of about $1000 \mathrm{G}$ at the level of the photosphere, and on the order of 100 times lower in the corona, depending on how the loop cross section changes. Rather than field strength, the Marshall Space Flight Center vector magnetograph group has shown over the years that the key to triggering flares is magnetic shear of loop arcades across a photospheric neutral line. As for field strength and X-ray emission of loops, Golub et al. proposed a scaling law 20 years ago as an adjunct to the Rosner, Tucker and Vaiana scaling law for pressure, temperature and length (Ap. J. 238, 343, 1980).

S. Berdyugina: It is known that flare time-scales are different for different types of magnetically active cool stars. What is your prediction for Be stars? 
B. Haisch: Without knowing what kind of magnetic field might be involved in Be flares, I would not know on what to base such a prediction. After all, Be stars are not supposed to flare at all.

M. Smith: I have two comments. (1) It is amazing to see how many processes have to operate successfully in tandem to produce what we so glibly refer to as a "flare." For these all to proceed through to completion must be a very infrequent occurrence even on an "active" Be star. It is tempting to associate something like this to Be outburstings because they too are infrequent. (2) The other comment is that magnetic interactions might also be important between the Be star and its disk (for $\gamma$ Cas this is a possible conjecture).

B. Haisch: One of the points I tried to make in my talk is that one can have a dynamo based on other kinds of instabilities, specifically a magneto-rotational instability that stresses the magnetic field penetrating a circumstellar disk. If that is what you mean by magnetic interactions involving a disk, I agree.

Ph. Stee: Do you know if it is really possible to apply solar theory to stars that are fast rotators (and can be distorted by the rotation) and that are not convective at their surface? Is it possible to keep loops (within the observed timescales) in a strong radiative wind?

B. Haisch: I think that the behaviour of rapidly-rotating RS CVn stars and the existence of a well-known correlation between rotation and X-ray luminosity is telling us that "solar theory" is relevant across a broad range of rotations. However when you get to apparently non-convective stars, I don't know what theory to apply. I also don't know whether coronal loops could survive within a radiatively-driven wind; I imagine you could have coronal loops with a wind flowing around them, but my imagination does not prove anything.

S. Owocki: Much of the flare ejection you show for the Sun is pressure driven by very hot gas. But many signatures of mass ejection on Be stars, e.g. high-velocity absorptions are still cool. What are the prospects to use magnetic "slingshots" to propel material upward in flares? On the Sun, aren't such ejections, e.g. prominences, typically at much lower speeds?

B. Haisch: I have a short description in my paper by Yokoyama and Shibata on these whip-like reconnections. If a lot of magnetic energy is released as heat, you see an X-ray jet on the Sun, but they point out that the same process can give rise to chromospheric-temperature (say 10000-20000 K) $\mathrm{H} \alpha$ surges. Both prominence eruptions and $\mathrm{H} \alpha$ surges on the Sun attain velocities in excess of $100 \mathrm{~km} \mathrm{~s}^{-1}$. I would not know how to scale this for Be stars, though, since we are back to the fundamental unknown about what kind of magnetic field might exist on these stars.

H. Henrichs: What physical mechanisms determine the decay of sunspot groups?

B. Haisch: I would say there are three major processes at work. First, diffusion driven by convective motions at the photosphere level. Indeed, this is the process that leads to the general polarity reversal of the solar dipole field. Secondly, magnetic reconnection involved in heating of coronal loops and flares would eliminate magnetic flux. The third factor is a bigger uncertainty: active regions develop and are sustained because of emergence of flux from below. At some point and for some reason that stops happening, and the sunspot group runs out of steam, so to speak. 